\title{
Decisive test of color coherence in proton-nucleus collisions at the LHC
}

\author{
Adam Bzdak ${ }^{1, *}$ and Vladimir Skokov ${ }^{2, \dagger}$ \\ ${ }^{1}$ RIKEN BNL Research Center, Brookhaven National Laboratory, Upton, NY 11973, USA \\ ${ }^{2}$ Department of Physics, Brookhaven National Laboratory, Upton, NY 11973, USA; \\ Department of Physics, Western Michigan University, Kalamazoo, MI 49008, USA
}

(Dated: September 14, 2018)

\begin{abstract}
Proton-nucleus collisions ( $\mathrm{p}+\mathrm{A})$ at LHC energies provide a rigorous test of the Color Glass Condensate (CGC), a model proposed to describe the high energy limit of Quantum Chromodynamics. In the CGC the average multiplicity of charged particles at midrapidity in $\mathrm{p}+\mathrm{A}$ collisions depends logarithmically on the number of participants, $N_{\text {part }}$. In contrast, the Wounded Nucleon Model (WNM) of independent nucleon-nucleon scatterings, verified at RHIC energies, predicts that multiplicity in p+A depends linearly on $N_{\text {part }}$. We argue that the dependence of mean multiplicity on $N_{\text {part }}$ in $\mathrm{p}+\mathrm{A}$ collisions at LHC energies can single out a model of particle production, thus offering a stringent test of the CGC and the WNM. Based on this observation we propose a novel experimental test of color coherence in $\mathrm{p}+\mathrm{A}$ collisions.

PACS numbers: 25.75.-q, 25.75.Ag
\end{abstract}

Understanding the properties of strongly interacting matter at extreme conditions is the ultimate goal of heavy-ion experiments at Relativistic Heavy-Ion Collider (RHIC) and the Large Hadron Collider (LHC). The theoretical description of nucleus-nucleus $(\mathrm{A}+\mathrm{A})$ collisions is complicated because of the importance of the nonperturbative nature of the strong forces at long distances, and the fact that one has to tackle a many body problem without obvious reduction to effective degrees of freedom. The last decade of collecting and analysing experimental data shows that a phenomenological application of hydrodynamics, an effective theory of long wavelength excitations in strongly-coupled systems, can successfully describe a large body of experimental data [1], including momentum spectra of produced particles and twoparticle correlations. However, from the theoretical perspective the application of hydrodynamics is not based on Quantum Chromodynamics (QCD) because many open issues are unresolved including the non-equilibrium early stage of heavy-ion collisions, thermalization, and many others. These problems demand development of the nonperturbative methods to $\mathrm{QCD}$, which are not available at present. Many models, including a holographic approach to QCD-like theories (see e.g. Ref. [2]) and the Color Glass Condensate (CGC) (see e.g. Ref. [3]) aspire to describe various stages of a heavy-ion collision. However, a commonly accepted and complete picture is still lacking.

The complexity of heavy-ion collisions is reduced considerably in the case of proton-proton $(\mathrm{p}+\mathrm{p})$ and protonnucleus $(\mathrm{p}+\mathrm{A})$ collisions owing to the expected dominance of the initial state effects [4], however recent studies of $\mathrm{p}+\mathrm{A}$ collisions [5-8] put this into question. The initial state effects are poorly studied in $\mathrm{A}+\mathrm{A}$ collisions with their strong final state interactions. Recent measurements at the LHC have provided new constraints on the models of particle production in $\mathrm{p}+\mathrm{p}$ and $\mathrm{p}+\mathrm{A}$ collisions, which can be used for further understanding of
A + A collisions. Especially, disentangling the initial and final state effects is of primary importance for many studies of QCD properties in A+A collisions, as the search for the QCD Critical Point, CP violation and extracting transport properties of quark-gluon plasma.

One of the most important questions in $\mathrm{p}+\mathrm{A}$ physics is whether the initial state of colliding nuclei behave as a superposition of its constituents or rather as a coherent gluon field predicted in the Color Glass Condensate. The CGC provides a framework to study particle production and scattering in QCD at high energies (small $x$ ). The key ingredients of the CGC are weak coupling, $\alpha_{s} \ll 1$; large gluon density $\propto 1 / \alpha_{s}$ corresponding to strong classical fields; nonlinear effects such as recombination and scattering of gluons characterized by single dynamically generated scale, $Q_{s}$, the so-called saturation momentum, a typical transverse momentum below which the field modes have large occupation numbers $\propto 1 / \alpha_{s}$.

At present, there is no direct experimental evidence in favor of the CGC. In this Letter, we provide a powerful yet simple method to disentangle the mechanism of particle production in $\mathrm{p}+\mathrm{A}$ collisions [29]. We show that in the CGC the mean number of produced particles at midrapidity depends logarithmically on the number of wounded nucleons, in a striking contrast to an expectation of a linear dependence on $N_{\text {part }}$. In experiments $N_{\text {part }}$ is difficult to measure in a model-independent way and we also propose an alternative probe based on the above observation, which can be straightforwardly measured at the LHC.

In the Wounded Nucleon Model (WNM) [10], the average multiplicity in $\mathrm{p}+\mathrm{A}$ collisions depends linearly on the number of participants

$$
\left\langle N_{\mathrm{ch}}^{\mathrm{pA}}\right\rangle=\frac{\left\langle n_{\mathrm{ch}}^{\mathrm{pp}}\right\rangle}{2} N_{\mathrm{part}},
$$

where $\left\langle N_{\mathrm{ch}}^{\mathrm{pA}}\right\rangle$ and $\left\langle n_{\mathrm{ch}}^{\mathrm{pp}}\right\rangle$ are the average numbers of pro- 


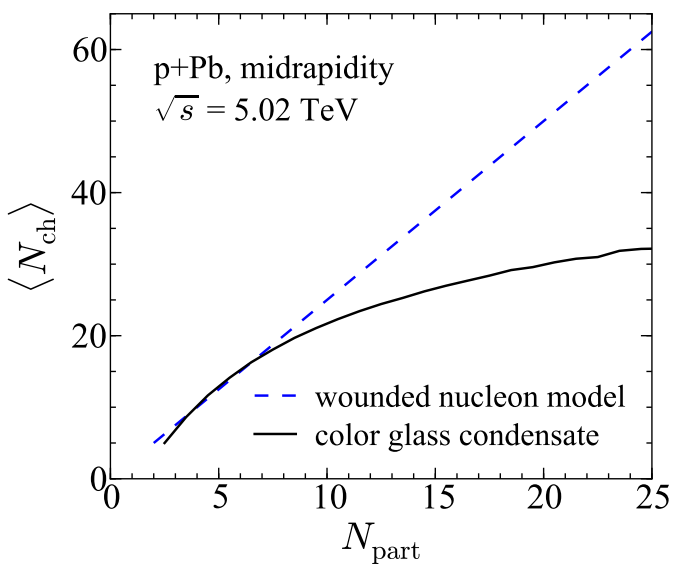

FIG. 1: The average number of particles at midrapidity, $\eta=$ 0 , in the Color Glass Condensate (CGC) and the Wounded Nucleon model (WNM) in $\mathrm{p}+\mathrm{Pb}$ collisions at $\sqrt{s}=5.02 \mathrm{TeV}$. In the CGC, the mean multiplicity depends logarithmically on the number of participants. In the WNM, average multiplicity is a linear function of $N_{\text {part }}$. In central p+A collisions, corresponding roughly to $N_{\text {part }}>18$, there is approximately a factor of 2 difference between the two models. The CGC curve is computed numerically in the rcBK model $[19,20]$.

duced particles in $\mathrm{p}+\mathrm{A}$ and $\mathrm{p}+\mathrm{p}$ collisions, respectively, and $N_{\text {part }}$ is the number of wounded nucleons. The interpretation of Eq. (1) is the following. Each participant in a nucleus is struck exactly once and produces a certain number of particles, which, on average, is equal to the half of that in $\mathrm{p}+\mathrm{p}$ collisions, $\left\langle n_{\mathrm{ch}}^{\mathrm{pp}}\right\rangle / 2$. The projectile proton is assumed to yield the same number of particles independently of how many interactions it underwent [30]. Equation (1) was experimentally verified for different energies, ranging from $10 \mathrm{GeV}$ [11] to 200 $\mathrm{GeV}$ in $\mathrm{d}+\mathrm{Au}$ collisions at RHIC [12]. Particularly successful is the description of $d+A u$ collisions: Eq. (1) was confirmed for the total number of produced particles [12] and their number at midrapidity [13]. Moreover, with an extension of the WNM, the entire pseudorapidity distribution at all measured centralities was described $[13,14]$.

It was formerly believed that multiplicity in $\mathrm{p}+\mathrm{A}$ depends on the number of collisions in accordance with

$$
\left\langle N_{\mathrm{ch}}^{\mathrm{pA}}\right\rangle=\left\langle n_{\mathrm{ch}}^{\mathrm{pp}}\right\rangle N_{\mathrm{coll}}=\left\langle n_{\mathrm{ch}}^{\mathrm{pp}}\right\rangle\left(N_{\mathrm{part}}-1\right) .
$$

In this scenario, each nucleon-nucleon collision in a nucleus produces on average $\left\langle n_{\mathrm{ch}}^{\mathrm{pp}}\right\rangle$ particles. In other words, a projectile proton creates new particles in every collision. Both Eqs. (1) and (2) are characterized by a linear dependence on the number of participants, although in the former case particle abundance is significantly lower. Equation (2) is expected to hold for the particles in the high transverse momentum region, $k_{\perp} \gg Q_{s}^{(\mathrm{A})}$ [31], where jets are produced in each nucleon-nucleon collision; while Eq. (1) is plausibly applicable for particles with transverse momenta in the non-perturbative region.

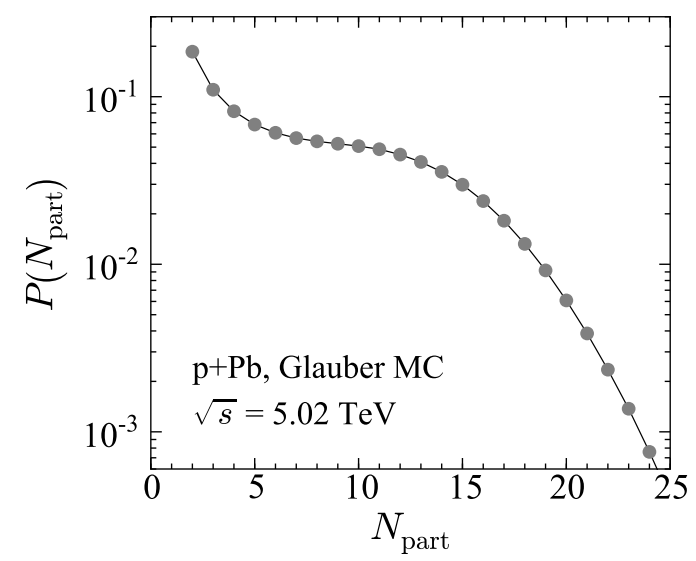

FIG. 2: The probability distribution of the number of wounded nucleons in $\mathrm{p}+\mathrm{Pb}$ collisions at $\sqrt{s}=5.02 \mathrm{TeV}$ in the Glauber Monte-Carlo model.

The CGC result in the non-perturbative regime is different from Eq. (1). On very general grounds it is predicted that at midrapidity [32]

$$
\left\langle N_{\mathrm{ch}}^{\mathrm{pA}}\right\rangle \sim \ln \left(N_{\mathrm{part}}\right) .
$$

We will not dwell on the technical aspects of the derivation of Eq. (3), but pass on at once to its intuitive understanding. The dependence (3) can be obtained from the integrated gluon transverse momentum spectrum found in Ref. $[15,16]$. This spectrum might be understood from the argumentation which follows. First of all, assuming validity of the CGC framework, we implicitly accept that $Q_{s}^{(\mathrm{p})}, Q_{s}^{(\mathrm{A})} \gg \Lambda_{\mathrm{QCD}}$. For the sake of argument, we also consider that the saturation momentum of a proton is much smaller then that of a nucleus, $Q_{s}^{(\mathrm{p})} \ll Q_{s}^{(\mathrm{A})}$. In this case, the gluon spectrum can be obtained analytically under reasonable assumptions. Gluons at large transverse momentum, $k_{\perp} \gg Q_{s}^{(\mathrm{A})}$, are affected by neither the field of the proton nor the nucleus, and consequently are produced according to the ordinary perturbation theory of QCD with the characteristic $1 / k_{\perp}^{4}$ dependence. The low $k_{\perp}$ gluons, $\Lambda_{\mathrm{QCD}}<k_{\perp}<Q_{s}^{(\mathrm{p})}$, are in the nonlinear regime with respect to both the fields of the proton and nucleus. The problem of gluon production in this domain is only tractable numerically. Here, the gluon distribution is expected to be independent of $k_{\perp}$ modulo logarithmic corrections. In the intermediate region, $Q_{s}^{(\mathrm{p})}<k_{\perp}<Q_{s}^{(\mathrm{A})}$, the proton's gluon field can be treated perturbatively, while that of the nucleus is in the saturation regime and must be accounted for classically. The gluon production can effectively be regarded as bremsstrahlung of large- $x$ partons in the classical gluon field of the nucleus. Thus, the transverse momentum dependence is modified from the perturbative one to the one inherently characteristic for bremsstrahlung $\sim 1 / k_{\perp}^{2}$ (see also Ref. [17] for the numerical confirmation of this dependence). 

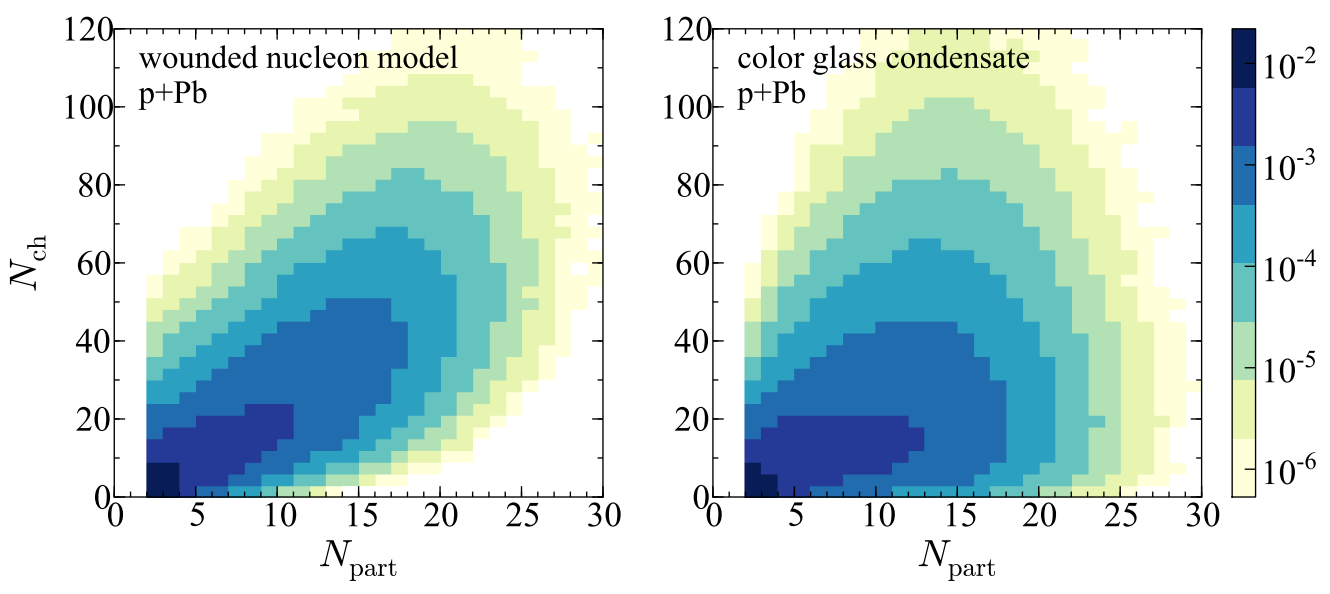

FIG. 3: The probability distribution of the number of participants, $N_{\text {part }}$, and the number of produced particles at midrapidity, $N_{\mathrm{ch}}$, in the Wounded Nucleon Model (left) and the Color Glass Condensate (right) in $\mathrm{p}+\mathrm{Pb}$ collisions at $\sqrt{s}=5.02$. For $N_{\text {part }}>10$, the CGC has a significantly larger fluctuations of $N_{\text {ch }}$ at a given number of $N_{\text {part }}$.

The gluon momentum distribution at midrapidity is dominated by this intermediate region $Q_{s}^{(\mathrm{p})}<k_{\perp}<Q_{s}^{(\mathrm{A})}$ with $d^{2} N / d^{2} k_{\perp} \sim 1 / k_{\perp}^{2}$ [33]. Integrating w.r.t. $k_{\perp}$, we obtain [34] [35]

$$
\left\langle N_{\mathrm{ch}}\right\rangle \sim \ln \left(\frac{Q_{s}^{(\mathrm{A})}}{Q_{s}^{(\mathrm{p})}}\right) \sim \ln \left(N_{\mathrm{part}}\right) .
$$

The second part of this equation, originates from the fact that $\left(Q_{s}^{(\mathrm{A})}\right)^{2}$ is proportional to the number of participants from the nucleus, $\left(Q_{s}^{(\mathrm{A})}\right)^{2} \propto N_{\text {part }}-1 \approx N_{\text {part }}$. This discussion is deficient in several aspects. More rigorous approach is to incorporate quantum corrections by solving renormalization group equations, as the JIMWLK hierarchy [18], on top of classical field defined by the ab-initio first principle calculations of the nuclear wave function at small $x$. The JIMWLK equations can be written in a closed form in the limit of large number of colors, the so called BK equation. The latter can be improved by taking into account the running coupling corrections. This approach was developed in Refs. [19, 20] and is referred to as rcBK in the literature. The initial conditions for the BK evolution are defined by the global fits of the deep inelastic scattering data performed by the AAMQS collaboration [21]. For more details of the rcBK implementation and its predictions for $\mathrm{p}+\mathrm{A}$ collisions we refer the reader to Refs. [19, 20].

Figure 1 illustrates the predictions of the CGC and the WNM for the average number of charged particles at midrapidity [36], $\eta=0$, in $\mathrm{p}+\mathrm{Pb}$ collisions at $\sqrt{s}=5.02$ $\mathrm{TeV}$. In peripheral collisions with $N_{\text {part }}=2$, corresponding to $\mathrm{p}+\mathrm{p}$, both models agree with the CMS measurement [22]. The rcBK results demonstrate expected logarithmic dependence on the number of participants. To assure that this is not an artefact of neglecting the higher order correlations in the JIMWLK evolution, the IP-
Glasma model [23] was used, which circumvents the problem of solving JIMWLK by parametrization of the saturation scale via the IP-Sat [24] model fitted to the HERA data. The IP-Glasma model reproduced the rcBK result with high precision [37]. We also verified Eq. (3) in the KLN model [25]. This agreement of different implementations of the CGC suggests that Eq. (3) is a universal property of the CGC.

As studied in Refs. [13, 26] both the WNM and CGC can describe the data on $\mathrm{d}+\mathrm{Au}$ collisions at RHIC. As seen from Fig. 1, the difference between the two models is noticeable in the region with high number of participants, $N_{\text {part }}>12$. We argue that this region is accessible at the LHC. Indeed, as demonstrated in Fig. 2, the probability distribution of $N_{\text {part }}$ in $\mathrm{p}+\mathrm{Pb}$ collisions is high even for $N_{\text {part }}=20$. This result is based on the standard Glauber Monte-Carlo model with the Gaussian inelastic nucleonnucleon profile $[14,27]$ and the inelastic cross section $\sigma=$ $70 \mathrm{mb}$.

It is a formidable problem to assess, in a modelindependent way, the number of wounded nucleons. Consequently, the observation presented in Fig. 1 may be challenging to test experimentally. Based on Fig. 1, one may speculate, that the WNM and the CGC should result in very different multiplicity distributions at high numbers of produced particles, $N_{\mathrm{ch}}$. This is because events with high $N_{\mathrm{ch}}$ are correlated with those having high number of participants, where the color coherence effects are best visible. However, this is not supported by direct calculations. As shown in Fig. 3, for a large number of participants, $N_{\text {part }}>10$, fluctuations of the number of produced particles at a given $N_{\text {part }}$ are significantly larger in the CGC than in the WNM [38]. Consequently the projected multiplicity distributions of the model predictions in Fig. 3 are approximately the same within the uncertainties of the models [39]. 


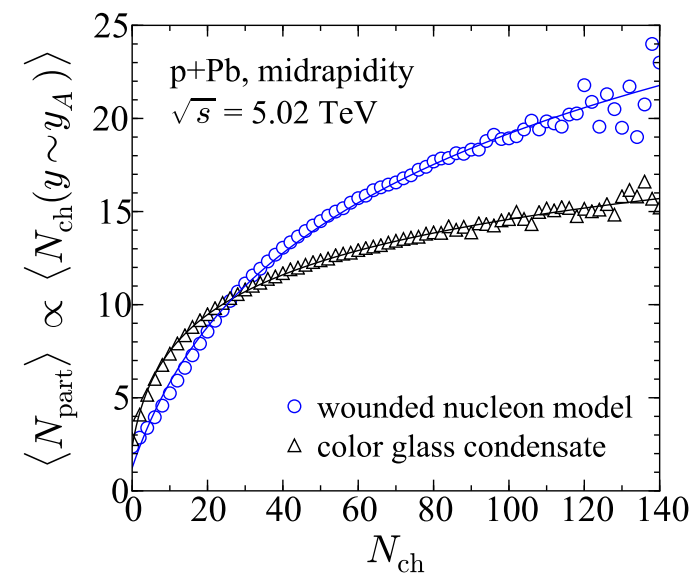

FIG. 4: The average number of participants, $\left\langle N_{\text {part }}\right\rangle$, at a given number of produced charged particles at midrapidity, $N_{\text {ch }}$, in the Wounded Nucleon Model and the Color Glass Condensate in $\mathrm{p}+\mathrm{Pb}$ collisions at $\sqrt{s}=5.02$. The average number of participants can be replaced by the mean number of particles in a (broad) vicinity of a nucleus fragmentation region, $\left\langle N_{\operatorname{ch}}\left(y \sim y_{A}\right)\right\rangle$, where $y_{A}$ is a nucleus rapidity (see the text for more details). Alternatively $\left\langle N_{\text {part }}\right\rangle$ could be estimated via the number of high $k_{\perp}$ particles (pions, photons etc.) that are known to scale with the number of collisions, which is equivalent to $N_{\text {part }}$ in $\mathrm{p}+\mathrm{A}$.

In this Letter we propose a novel approach to discriminate between the CGC and the WNM. In Fig. 4 we present the relation between the average number of participants at a given number of produced charged particles at midrapidity. The CGC and the WNM lead to very different relations especially for low and large number of produced particles. For example, at $N_{\mathrm{ch}}=140$ the mean number of participants differs approximately by $50 \%$, being larger in the WNM than in the CGC. At $N_{\mathrm{ch}}=10$ the situation is opposite with more participants, on average, in the CGC than the WNM. The latter can be deduced from Figs. 1 and 3. Indeed, in the CGC, events with a broad range of $N_{\text {part }}$ can deliver a small number of particles, wherein in the WNM only events with small $N_{\text {part }}$ can yield small $N_{\text {ch }}$. At large $N_{\text {ch }}$, strong fluctuations in the CGC can lead to a large number of produced particles even if $N_{\text {part }}$ is not particularly large.

One could argue that $\left\langle N_{\text {part }}\right\rangle$ is as difficult to measure as $N_{\text {part }}$, which is, however, not the case. The mean number of participants at a given $N_{\mathrm{ch}}$ at zero (pseudo)rapidity can be extracted in several ways. Below, we list them in descending order of theoretical reliability and experimental accessibility.

1. Number of charged particles in a vicinity of the nucleus fragmentation region, $d N /\left.d \eta\right|_{\eta \sim \eta_{A}}$. For sufficiently high (pseudo)rapidity it is proportional to $\left(N_{\text {part }}-1\right)$ modulo small corrections. This observable can be further improved by considering the difference $\left(d N /\left.d \eta\right|_{\eta=\eta^{*}}-d N /\left.d \eta\right|_{\eta=-\eta^{*}}\right)$, in which central rapidity effects cancel out. We checked that the ratio of this combination to $\left\langle N_{\text {part }}\right\rangle$ is independent of $N_{\text {ch }}$ in the rcBK model already at the moderate values of $\eta^{*} \geq 2$.

2. It is known that at large $k_{\perp}$ particles scale with the number of collisions, $N_{\text {coll }}=N_{\text {part }}-1 \mathrm{in} \mathrm{p}+\mathrm{A}$. This is the case in the CGC, where $R_{\mathrm{pPb}}=1$ [20]. Thus the average number of participants in Fig. 4 can be replaced by the number of high $k_{\perp}$ particles.

3. The observable proposed in 2), can also be applied to high $k_{\perp}$ photons.

In conclusion, we argued that $\mathrm{p}+\mathrm{A}$ collisions at the LHC have potential to produce the evidence for the Color Glass Condensate. The CGC predicts a logarithmic dependence of the midrapidity average number of produced particles on the number of participants (wounded nucleons). This is a direct manifestation of the color coherence effects present in the CGC. As we demonstrated, in central $\mathrm{p}+\mathrm{Pb}$ collisions the mean number of particles at midrapidity in the CGC is expected to be smaller approximately by a factor of 2 compared to predictions of the Wounded Nucleon Model, where the mean number of particles depends linearly on $N_{\text {part }}$. Similar dependence is expected to be present in all models based on the Glauber initial conditions disregarding color coherence effects. Based on this observation we proposed a novel and straightforward measurement, which can be carried out at the LHC.

We thank Adrian Dumitru, Larry McLerran and Al Mueller for valuable comments and in-depth discussions. Comments by Robert Pisarski, Bjoern Schenke, Paul Sorensen and Raju Venugopalan are acknowledged. A.B. is supported through the RIKEN-BNL Research Center. V.S. is supported by the U.S. Department of Energy under contract \#DE-AC02-98CH10886.

Note added. - After submission of our paper to Physical Review Letters, preliminary data were presented by the ATLAS Collaboration on $\mathrm{p}+\mathrm{Pb}$ collisions [28]. The dependence of the number of charged particles on the number of participants appears to be close to linear, suggesting that the WNM is applicable at LHC energies. This conclusion is sensitive, however, to how the number of participants is extracted in $\mathrm{p}+\mathrm{Pb}$ collisions.

* Electronic address: ABzdak@bnl.gov

$\dagger$ Electronic address: VSkokov@quark.phy.bnl.gov

[1] W. Florkowski, Phenomenology of Ultra-Relativistic Heavy-Ion Collisions (World Scientific Publishing Company, Singapore, 2010).

[2] O. DeWolfe, S. S. Gubser, C. Rosen and D. Teaney, arXiv:1304.7794 [hep-th]. 
[3] Y. V. Kovchegov and E. Levin, Quantum Chromodynamics at High Energy (Cambridge University Press, 2012); F. Gelis, Int. J. Mod. Phys. A 28, 1330001 (2013).

[4] K. Dusling and R. Venugopalan, Phys. Rev. D 87, 094034 (2013).

[5] P. Bozek, Phys. Rev. C 85, 014911 (2012).

[6] P. Bozek and W. Broniowski, Phys. Rev. C 88, 014903 (2013).

[7] A. Bzdak, B. Schenke, P. Tribedy and R. Venugopalan, Phys. Rev. C 87, 064906 (2013).

[8] A. Bzdak and V. Skokov, Phys. Lett. B 726, 408 (2013).

[9] D. Kharzeev and M. Nardi, Phys. Lett. B 507, 121 (2001).

[10] A. Bialas, M. Bleszynski and W. Czyz, Nucl. Phys. B 111, 461 (1976).

[11] J. E. Elias et al., Phys. Rev. D 22, 13 (1980).

[12] B. B. Back et al. [PHOBOS Collaboration], Phys. Rev. C 72, 031901 (2005).

[13] A. Bialas and W. Czyz, Acta Phys. Polon. B 36, 905 (2005).

[14] A. Bialas and A. Bzdak, Phys. Rev. C 77, 034908 (2008); Acta Phys. Polon. B 38, 159 (2007).

[15] Y. V. Kovchegov and A. H. Mueller, Nucl. Phys. B 529, 451 (1998).

[16] A. Dumitru and L. D. McLerran, Nucl. Phys. A 700, 492 (2002).

[17] J. P. Blaizot, F. Gelis and R. Venugopalan, Nucl. Phys. A 743, 13 (2004).

[18] I. Balitsky, Nucl. Phys. B 463, 99 (1996); J. JalilianMarian, A. Kovner, A. Leonidov and H. Weigert, Phys. Rev. D 59, 014014 (1998).

[19] J. L. Albacete and A. Dumitru, arXiv:1011.5161.

[20] J. L. Albacete, A. Dumitru, H. Fujii and Y. Nara, Nucl. Phys. A 897, 1 (2013).

[21] J. L. Albacete, N. Armesto, J. G. Milhano, P. QuirogaArias and C. A. Salgado, Eur. Phys. J. C 71, 1705 (2011).

[22] V. Khachatryan et al. [CMS Collaboration], Phys. Rev. Lett. 105, 022002 (2010).

[23] B. Schenke, P. Tribedy and R. Venugopalan, Phys. Rev. Lett. 108, 252301 (2012).

[24] H. Kowalski and D. Teaney, Phys. Rev. D 68, 114005 (2003).

[25] A. Dumitru, D. E. Kharzeev, E. M. Levin and Y. Nara, Phys. Rev. C 85, 044920 (2012).

[26] D. Kharzeev, E. Levin and M. Nardi, Nucl. Phys. A 730,
448 (2004) [Erratum-ibid. A 743, 329 (2004)].

[27] M. Rybczynski and W. Broniowski, Phys. Rev. C 84, 064913 (2011).

[28] E. Shulga for the ATLAS Collaboration, talk at the International Conference on the Initial Stages in High-Energy Nuclear Collisions, Illa de A Toxa, Spain, 2013.

[29] It should be noted that A+A collisions are not very effective in discriminating between different models of particle production, see e.g., [9].

[30] This is justified for particles with relatively small transverse momenta, because their formation time is longer than the time needed for a proton to pass through a nucleus. See Ref. [14] for more details.

[31] Here $Q_{s}^{(\mathrm{A})}$ is the saturation momentum of a nucleus.

[32] An analogous dependence was derived for $\mathrm{A}+\mathrm{A}$ collisions in Ref. [9]: $\left\langle N_{\text {ch }}\right\rangle \sim N_{\text {part }} \ln N_{\text {part }}$.

[33] The gluon fragmentation function is not expected to modify the conclusions of this article, which are drawn for the charged particle multiplicity.

[34] It should be noted that the different dependence of $\left\langle N_{\mathrm{ch}}\right\rangle$ on $N_{\text {part }}$ in the WNM and CGC leads to different values of $\left\langle N_{\text {part }}\right\rangle$ in a given range of the measured number of particles at midrapidity. Thus, to extract $\left\langle N_{\text {part }}\right\rangle$ reliably one has to perform measurements near the nucleus rapidity, where the number of particles depends linearly on $N_{\text {part }}$ in both models.

[35] See also Ref. [16], where logarithmic corrections were taken into account.

[36] It should be noted that the jet contribution can slightly modify the CGC prediction. The projectile proton interacts many times with the nucleons in a nucleus and an appropriate $N_{\text {coll }}$ term should be added (however, all nucleons in a nuclei are struck only once and for them such term is not warranted). This would result in a slightly faster increase of $\left\langle N_{\mathrm{ch}}\right\rangle$ with $N_{\text {part }}$. We do not expect this contribution to be larger than $10 \%$ and we neglect it in this article.

[37] We thank Bjoern Schenke for providing us with the IPGlasma results.

[38] In both models we generated $5 \times 10^{6}$ events.

[39] In the WNM each participant populates particles according to the negative binomial distribution. We use $\left\langle n_{\mathrm{ch}}^{\mathrm{pp}}\right\rangle=5$ and $k_{\mathrm{pp}}=1.1$. 